\section{La planaire : un ver immortel pour élucider la réponse immunitaire de l'homme}

CNRS UMR 7278, IRD198, Inserm U1095,

Aix-Marseille université, URMITE, faculté de médecine,

27, boulevard Jean Moulin, 13385 Marseille Cedex 05,

France.

eric.ghigo@univ-amu.fr
> Les microbes (parasites, virus, bactéries) ont une distribution mondiale; de ce fait, tous les animaux vertébrés et invertébrés y sont exposés. Certains de ces microbes sont inoffensifs, d'autres sont des agents pathogènes; il faut donc aux animaux un système de protection (le système immunitaire) pour lutter contre les agents infectieux et survivre. La connaissance et la compréhension des interactions entre les microbes et leurs hôtes sont d'un intérêt médical considérable. L'utilisation des organismes modèles invertébrés comme la mouche Drosophila melanogaster ou le nématode Caenorhabditis elegans, a conduit à des découvertes importantes dans le domaine des interactions hôtepathogène $[1,2]$. Ces organismes ont été choisis pour la connaissance de leurs génomes, la possibilité d'y invalider ou d'y surexprimer certains gènes, ainsi que pour leur temps de génération court et leur facilité d'utilisation. Cependant, une des caractéristiques de ces organismes est qu'ils sont sensibles à de nombreux agents pathogènes pour l'homme. Il paraît logique de penser qu'un organisme qui a développé une résistance élevée vis-à-vis d'agents pathogènes humains pourrait aider à une meilleure connaissance de la réponse immunitaire de l'homme; c'est le cas de la planaire, comme le démontrent nos travaux, publiés récemment dans le journal Cell Host and Microbe [3].

\section{La planaire}

Les planaires sont des vers plats aquatiques non parasitaires appartenant au phylum des Plathelminthes, connus pour leur extraordinaire capacité à régénérer n'importe quelle partie endommagée de leur corps. Cette capacité va encore plus loin puisque les planaires peuvent reformer une nouvelle tête après décapitation, et même un ver entier à partir de n'importe quel fragment de leur corps [4]. Thomas Morgan avait démontré en 1898 que chaque fragment issu d'une planaire découpée en 276 morceaux donnait naissance par régénération à une nouvelle planaire [5]. Il est maintenant établi que ce processus de régénération requiert une population de cellules appelées «néoblastes » qui sont des cellules souches pluripotentes adultes [6]. Ces néoblastes vont assurer la régénération de n'importe quel tissu ou organe de la planaire. Avec la découverte de I'ARN interférence et les avancées en matière de séquençage du génome, les planaires des espèces Schmidtea mediterranea et Dugesia japonica ont prouvé qu'elles représentaient de puissants systèmes d'étude des mécanismes complexes de la régénération tissulaire et du rôle des cellules souches dans la réponse mise en place lors d'une lésion $[7,8]$.

La réponse immunitaire de la planaire Jusqu'à aujourd'hui, tous les travaux de recherche chez la planaire se sont concentrés sur l'analyse des mécanismes de régénération, cependant aucun travail n'avait cherché à définir sa réponse immunitaire. L'étude de cette réponse peut s'avérer contributive, puisque ce ver est en contact permanent avec des bactéries. En effet, dans la nature, les planaires sont des prédateurs et des charognards, se nourrissant de larves de moustiques et de détritus, ce qui requiert une tolérance importante face à une grande diversité et une grande quantité de microbes ingérés par inadvertance.

Pour la première fois, la réponse immunitaire de la planaire, plus précisément de l'espèce $D$. japonica a été étudiée, et le modèle planaire établi comme système d'analyse des interactions hôte-pathogène [3]. Les planaires ont été inoculées avec des bactéries pathogènes pour l'homme et les organismes modèles classiques comme le nématode et la drosophile, telles que Mycobacterium tuberculosis, Staphyloccocus aureus ou encore Legionella pneumophila (16 souches de ces pathogènes testées). II s'est avéré que la planaire était capable de résister à l'infection par ces bactéries, même avec des inoculum élevés. Une fois ingérées, les bactéries se localisent dans les cellules phagocytaires, dans de larges vacuoles au sein du système gastro-vasculaire de la planaire. Après une semaine, les bactéries sont totalement éliminées du ver, quelle que soit leur virulence. La planaire est donc très résistante à l'infection bactérienne, contrairement à l'homme et aux autres organismes modèles. Afin de caractériser la réponse immunitaire de la planaire, nous avons déterminé le profil d'expression génique, par séquençage des ARN lors de l'infection par S. aureus et $L$. pneumophila, ce qui a permis d'identifier un grand nombre de gènes surexprimés lors de l'infection. Dix pour cent d'entre eux ont été invalidés lors d'un criblage par ARN interférence, ce 

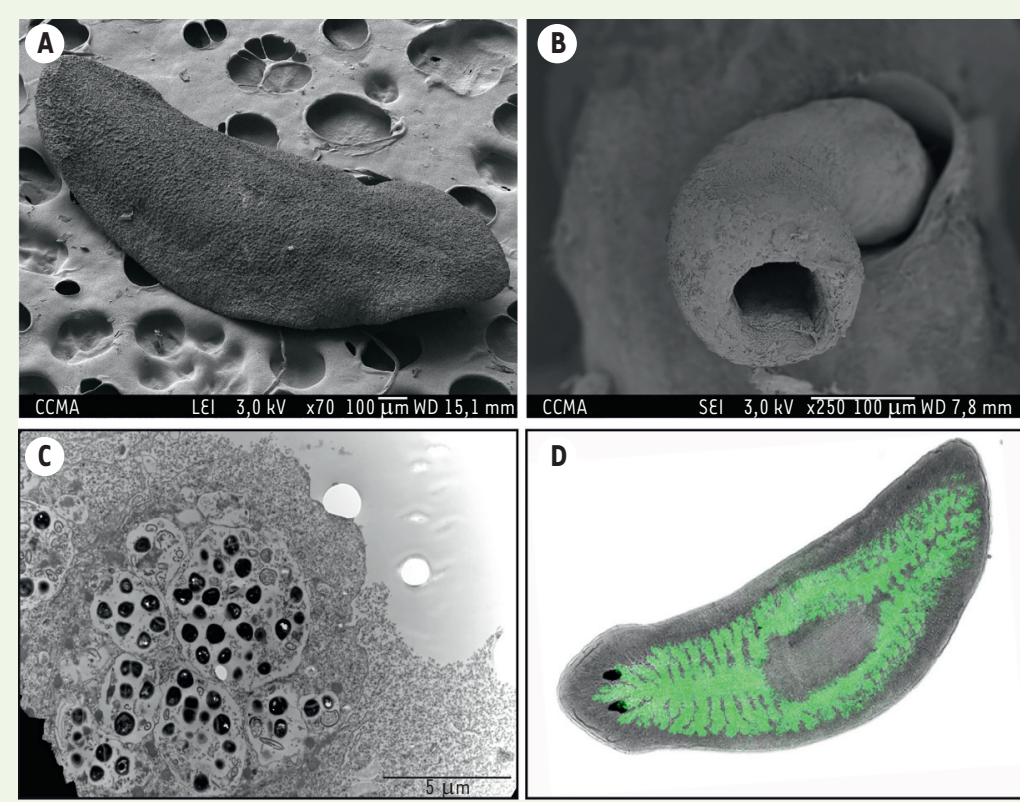

Figure 1. La planaire: un modèle d'étude des interactions hôte-pathogène. A. La planaire de l'espèce $D$. japonica observée en microscopie électronique à balayage. B. Pharynx de la planaire. C. Planaire infectée par la bactérie L. pneumophila marquée au FITC (vert). D. Cellules de la planaire infectées par $S$. aureus observées en microscopie électronique à transmission.

qui a permis d'identifier 18 gènes requis pour la clairance bactérienne. Huit de ces 18 gènes sont requis pour que s'exprime une résistance à l'infection par les bactéries à Gram négatif, Gram positif ou par les mycobactéries. Parmi ces 18 gènes, 3 ont des orthologues chez les mammifères et plus particulièrement chez l'homme: ils codent pour la phosphatase DUSP19 (dual specificity protein phosphatase 19), le modulateur de la voie de signalisation de l'insuline PAQR3 (progestin and adipol receptor family member III), et MORN2 (membrane occupation and recognition nexus 2).

\section{De la planaire à l'homme}

Le gène MORN2 s'est avéré être le plus intéressant à étudier pour plusieurs raisons : (1) il a été perdu au cours de l'évolution par les lignées menant à la drosophile $D$. melanogaster et au nématode C. elegans; (2) l'analyse des transcriptomes publiquement accessibles révèle que MORN2 est très fortement exprimé dans les cellules immunitaires humaines (macrophages, monocytes, lymphocytes, cellules natural killer), mais aussi par les cellules qui sont les premières à rencontrer les microbes, comme les cellules épithéliales trachéales, nasales et pulmonaires; (3) il n'y a aucune information sur le rôle fonctionnel de MORN2. Le gène MORN2 a été surexprimé dans des macrophages humains infectés par L. pneumophila, S. aureus ou M. tuberculosis. Les macrophages humains deviennent alors capables d'éliminer ces bactéries. A contrario, et de façon très intéressante, dans les macrophages invalidés pour MORN2, les bactéries se multiplient. Des expériences de biologie cellulaire et de biochimie révèlent que MORN2 agit probablement en induisant la phagocytose dépendante de LC3 (LC3-associated phagocytosis ou LAP), une protéine de l'autophagie qui potentialise la destruction des bactéries internalisées dans un phagolysosome $[9,10]$. II est intéressant de noter que la surexpression de MORN2 issue de la planaire dans les macrophages humains réduit la survie des agents pathogènes testés, suggérant une origine ancienne du mécanisme de la phagocytose associée à LC3.

\section{Conclusion et perspectives}

Ce travail démontre que la planaire, du fait de sa haute résistance aux agents pathogènes pour l'homme, est un bon modèle pour l'étude et la compréhension de l'évolution de la réponse immune humaine. Les molécules identifiées pourraient être de nouvelles cibles thérapeutiques dans la lutte contre la tuberculose, la légionellose ou le staphylocoque doré. Par ailleurs, ce travail démontre que l'approfondissement de nos connaissance des systèmes biologiques passe par la recherche et l'exploitation de modèles atypiques. $\diamond$ Planaria: an immortal worm to clarify human immune response

\section{LIENS D'INTÉRÊT}

Les auteurs déclarent n'avoir aucun lien d'intérêt concernant les données publiées dans cet article.

\section{REMERCIEMENTS}

Ce travail à été financé par le CNRS (PEPS 2010 ع. Ghigo).

\section{RéFÉRENCES}

1. Lemaitre B, Hoffmann J. The host defense of Drosophila melanogaster. Annu Rev Immunol 2007 ; $25: 697-743$.

2. Irazoqui JE, Urbach JM, Ausubel FM. Evolution of host innate defence: insights from Caenorhabditis elegans and primitive invertebrates. Nat Rev Immunol 2010 ; $10: 47-58$.

3. Abnave P, Mottola G, Gimenez G, et al. Screening in planarians identifies MORN2 as a key component in LC3-associated phagocytosis and resistance to bacterial infection. Cell Host Microbe 2014 ; 16 : 338-50.

4. Elliott SA, Sanchez Alvarado A. The history and enduring contributions of planarians to the study of animal regeneration. Wiley Interdisciplinary Reviews $2012 ; 2$ : 301-26.

5. Morgan TH. Experimental studies of the regeneration of Planaria maculata. Archiv für Entwicklungsmechanik der Organismen $1898 ; 7: 364-97$.

6. Wagner DE, Wang IE, Reddien PW. Clonogenic neoblasts are pluripotent adult stem cells that underlie planarian regeneration. Science $2011 ; 332$ : 811-6.

7. Sanchez Alvarado A, Newmark PA, Robb SM, Juste R. The Schmidtea mediterranea database as a molecular resource for studying platyhelminthes, stem cells and regeneration. Development $2002 ; 129$ : 5659-65. 


\section{RÉFÉRENCES}

8. Rink JC. Stem cell systems and regeneration in planaria. Dev Genes Evol 2013 ; 223 : 67-84.
9. Martinez J, Almendinger J, Oberst A, et al.

Microtubule-associated protein 1 light chain 3 alpha (LC3)-associated phagocytosis is required for the efficient clearance of dead cells. Proc Natl Acad Sci USA 2011 ; 108 : 17396-401.

\section{NOUVELle}

\section{Rôle clé des Treg CD8* spécifiques d'un allopeptide en transplantation}

Élodie Picarda, Jason Ossart, Séverine Bézie, Carole Guillonneau
10. Joubert PE, Pombo Grégoire I, Meiffren G, et al. Autophagie et pathogènes «Bon appétit Messieurs! ». Med Sci (Paris) $2011 ; 27: 41-7$.
Inserm UMR1064 - centre de recherche en transplantation et immunologie ITUN, centre hospitalier universitaire de Nantes, faculté de médecine, université de Nantes, 30, boulevard Jean Monnet, 44093, Nantes Cedex 01, France. carole.guillonneau@univ-nantes.fr

\section{Les Treg CD8 ${ }^{+}$CD45RC low}

en transplantation : rôle et mécanismes d'action

Des études phénotypiques et fonctionnelles récentes ont permis de distinguer plusieurs sous-populations de Treg $\mathrm{CD}^{+}$chez la souris, le rat et l'Homme. Une population en particulier, les Treg naturels (naïfs) de type $C D 8^{+} C D 45 R C^{\text {low }}$ a été identifiée chez le rat en 2004 [6]. Dans un modèle d'allotransplantation cardiaque incompatible chez le rat, nous avons montré pour la première fois que le blocage de la voie de costimulation CD40/CD40L L (via un adénovirus codant pour la molécule CD40Ig empêchant l'interaction ligand-récepteur) induit une survie indéfinie de l'allogreffe. Le mécanisme met en jeu la génération in vivo de Treg CD8 ${ }^{+} C D 45 R C^{\text {low }}$ spécifiques d'antigènes du donneur ( $a T r e g\left(D 8^{+} C D 45 R C^{\text {low }}\right.$ ) et dont le répertoire est biaisé en faveur de l'expression de la chaîne V $\beta 11$ du TCR $[4,5]$. Contrairement aux Treg naïfs, les aTreg CD8 ${ }^{+}$CD45RC ${ }^{\text {low }}$ sont capables de transférer la tolérance à des receveurs secondaires naïfs. Nous avons décrit

\footnotetext{
${ }^{2}$ CD40 est un membre de la superfamille des récepteurs du TNF. Il est exprimé à la surface des lymphocytes B et des CPA. CD40 interagit avec son ligand, CD40L (CD154), exprimé sélectivement à la surface des lymphocytes $T$ activés, et cette interaction est indispensable à la coopération entre lymphocytes B et lymphocytes T.
} 